\title{
A STABILISED TOTAL LAGRANGIAN CORRECTED SMOOTH PARTICLE HYDRODYNAMICS TECHNIQUE IN LARGE STRAIN EXPLICIT FAST SOLID DYNAMICS
}

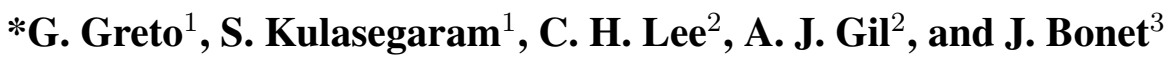 \\ ${ }^{1}$ Cardiff University \\ The Parade, Cardiff, CF24 3AA \\ e-mail: \{gretog,kulasegarams\}@ cardiff.ac.uk \\ ${ }^{2}$ Swansea University \\ Bay Campus, Swansea, SA1 8EN \\ \{a.j.gil,c.h.lee\}@swansea.ac.uk \\ ${ }^{3}$ University of Greenwich \\ London, SE10 9LS \\ j.bonet@greenwich.ac.uk
}

Keywords: Conservation laws, SPH, Locking, Incompressibility, Fast dynamics, JST.

\begin{abstract}
An explicit Total Lagrangian mixed momentum/strains formulation [1-5], in the form of a system of first order conservation laws, has been recently proposed to overcome the shortcomings posed by the traditional second order displacement-based formulation, namely: (1) bending and volumetric locking difficulties; (2) hydrostatic pressure fluctuations; and (3) reduced order of convergence for derived variables. Following the work of Bonet and Kulasegaram [6] 7], the main objective of this paper is the adaptation of Corrected Smooth Particle Hydrodynamics (CSPH) in the context of Total Lagrangian mixed formulation. Appropriate nodally conservative Jameson-Schmidt-Turkel (JST) stabilisation is introduced by taking advantage of the conservation laws. This mixed linear momentum-deformation gradient technique performs extremely well in nearly incompressible bending dominated scenarios [1, 2] without the appearance of spurious pressure oscillations. Additionally, as both linear momentum and deformation gradient are used as primary variables of the system, equal order of approximation should be achieved in both fields. A series of numerical examples are carried out to assess the applicability and robustness of the proposed algorithm.
\end{abstract}




\section{INTRODUCTION}

Dynamic explicit displacement-based finite element codes based on low order finite element technology are commonly used for complex numerical simulations in the aerospace, automotive, biomedical, defence and manufacturing fields. However, difficulties arise in modelling high speed impacts or materials capable of enduring large finite deformations before failing. Most computational codes prefer to employ the 8-noded underintegrated hexahedral element to model solid components. Many practical applications (i.e. crashworthiness and drop-impact modelling), however, experience extremely large solid deformations accompanied by severe mesh distortion. This may lead to poorly shaped elements, unless some form of adaptive remeshing is applied.

From the viewpoint of spatial discretisation, the standard displacement based formulation with low order elements is known to experience locking behaviour in nearly-incompressible, bending dominated scenarios. It is also known that the use of linear interpolation within a finite element leads to second order convergence for the primary variables, but one order less for derived variables. From the time discretisation point of view, the Newmark method has a tendency to introduce high frequency noise in the solution field, especially in the vicinity of sharp spatial gradients.

In order to address these issues, a new mixed-formulation for Total Lagrangian fast solid dynamics has recently been proposed [4]. The new mixed-formulation consists in a system of first order conservation laws, formally akin to Computational Fluid Dynamics (CFD) models, with linear momentum and deformation gradient being the unknown conservation variables. In [2], the technique was applied in the framework of Finite Volume method, incorporating a Jameson-Schmidt-Turkel (JST) stabilising contribution [8]. Adding JST has the dual aim of (a) accurately representing potential discontinuities in the solution, and (b) introducing artificial dissipation to it where continuous. In [3], stabilisation is achieved through the use of Petrov-Galerkin algorithm. All these attempts have yielded promising results in two- and threedimensional applications, with velocities (or displacements) and stresses (or strains) achieving the same degree of accuracy.

The main goal of this paper is the introduction of a stabilised Corrected Smooth Particle Hydrodynamics (CSPH) methodology, tailor-made for the aforementioned mixed formulation. The adoption of a system of first order conservation laws allows CFD techniques to be introduced within the context of particle methods for solid mechanics, in the form of an adapted nodally conservative JST stabilisation [2]. Incorporation of JST stabilisation will be useful to provide artificial (numerical) dissipation and shock-capturing capabilities. In particular, the second order (harmonic) and fourth order (biharmonic) operators employed in the JST stabilisation can be readily obtained by closed-form differentiation of the interpolating kernel functions. A quintic kernel approximation [6] is suitably used, due to the presence of the biharmonic JST operator.

This paper is structured as follows: section 2 outlines the theoretical framework of the $\boldsymbol{p}-\boldsymbol{F}$ mixed formulation system of equations; section 3 focuses on SPH spatial discretisation of the governing equations. After that, section 4 describes the Total Variation Diminishing RungeKutta (TVD-RK) time integration method, used in the numerical computations. A series a numerical examples in section 5 assesses the feasibility and robustness of the proposed formulation. Concluding remarks are summarised in section 6. 


\section{2 p-F MIXED METHODOLOGY IN REVERSIBLE ELASTODYNAMICS}

A deformable continuum body, of initial shape and position described by $\boldsymbol{X} \in V \subset \mathbb{R}^{3}$, finds itself in a current (or deformed) configuration $\boldsymbol{x} \in V(t) \subset \mathbb{R}^{3}$ at the end of a motion $\Phi$ that links each material particle in $\boldsymbol{X}$ to its current position $\boldsymbol{x}$ at time instant $t, \boldsymbol{x}=\boldsymbol{\Phi}(\boldsymbol{X}, t)$, see fig. 1a,

Neglecting dissipative effects, the dynamics of motion $\boldsymbol{\Phi}$ can be described by a $\boldsymbol{p}-\boldsymbol{F}$ mixed formulation system of first order conservation laws as [1,2]:

$$
\left\{\begin{aligned}
\frac{\partial \boldsymbol{p}}{\partial t}-\boldsymbol{\nabla}_{0} \cdot \boldsymbol{P}(\boldsymbol{F}) & =\rho_{0} \boldsymbol{b} \\
\frac{\partial \boldsymbol{F}}{\partial t}-\boldsymbol{\nabla}_{0} \cdot\left(\frac{1}{\rho_{0}} \boldsymbol{p} \otimes \boldsymbol{I}\right) & =\mathbf{0}
\end{aligned}\right.
$$

Here, $\boldsymbol{p}\left(=\rho_{0} \boldsymbol{v}\right)$ is the linear momentum per unit of undeformed volume, $\rho_{0}$ is the material density, $\boldsymbol{v}$ represents the velocity field, $\boldsymbol{P}$ is the first Piola-Kirchhoff stress tensor, $\boldsymbol{b}$ is the body force per unit mass, $\boldsymbol{F}$ is the deformation gradient and $\boldsymbol{I}$ is the identity matrix. The notation $\boldsymbol{\nabla}_{0}$ denotes the material gradient operator (in undeformed space), where $\left[\nabla_{0}\right]_{I} \equiv \frac{\partial}{\partial X_{I}}$. The first equation in (1) represents the conservation of linear momentum, while the second equation in (1) is a conservation law for the deformation gradient $\boldsymbol{F}$. The above laws (1) can be written in a more compact form:

$$
\frac{\partial \mathcal{U}}{\partial t}+\frac{\partial \mathcal{F}_{I}}{\partial X_{I}}=\mathcal{S} \quad \forall I=1,2,3
$$

where $\mathcal{U}$ is the vector of conservation variables, $\mathcal{F}_{I}$ is the flux vector in the material direction $I=1,2,3$ and $\mathcal{S}$ is the source term described by:

$$
\mathcal{U}=\left[\begin{array}{c}
\boldsymbol{p} \\
\boldsymbol{F}
\end{array}\right] \quad \mathcal{F}_{\boldsymbol{N}}=\mathcal{F}_{I} N_{I}=\left[\begin{array}{c}
-\boldsymbol{P} \boldsymbol{N} \\
-\frac{1}{\rho_{0}} \boldsymbol{p} \otimes \boldsymbol{N}
\end{array}\right] \quad \mathcal{S}=\left[\begin{array}{c}
\rho_{0} \boldsymbol{b} \\
\mathbf{0}
\end{array}\right]
$$

For closure of the above system (1), a material constitutive law that describes the way in which $\boldsymbol{P}$ depends on $\boldsymbol{F}$ has to be introduced. For this purpose, a standard nearly incompressible hyperelastic NeoHookean model will be used below. The path-independent behaviour of a hyperelastic material enables the direct extraction of the first Piola-Kirchhoff stress $\boldsymbol{P}$ from a strain energy functional $\psi(\boldsymbol{F}, J)$ as

$$
\boldsymbol{P}=\frac{\partial \psi}{\partial \boldsymbol{F}}
$$

where $J$ is the Jacobian of the deformation gradient, $J=\operatorname{det} \boldsymbol{F}$.

It is useful to decompose the strain energy functional $\psi$ into the summation of a volumetric and a deviatoric component:

$$
\psi=\psi^{\mathrm{vol}}+\psi^{\mathrm{dev}}
$$

where

$$
\psi^{\mathrm{vol}}=\frac{1}{2} \kappa(J-1)^{2} \quad \psi^{\mathrm{dev}}=\frac{1}{2} \mu\left(J_{\boldsymbol{F}}^{-2 / 3}(\boldsymbol{F}: \boldsymbol{F})-3\right)
$$

in (6), $\kappa$ is the bulk modulus of the material, and $\mu$ is its shear modulus. 
The first Piola-Kirchhoff stress tensor $\boldsymbol{P}$ can then also be decomposed into the summation of deviatoric, $\boldsymbol{P}_{d e v}$, and volumetric, $\boldsymbol{P}_{v o l}$, contributions:

$$
\boldsymbol{P}=\boldsymbol{P}_{d e v}+\boldsymbol{P}_{v o l} \quad \boldsymbol{P}_{v o l}=p \boldsymbol{H} \quad \boldsymbol{P}_{d e v}=\mu J^{-2 / 3}\left(\boldsymbol{F}-\frac{1}{3}(\boldsymbol{F}: \boldsymbol{F}) \boldsymbol{F}^{-T}\right)
$$

in (7), $p=\kappa(J-1)$ is the hydrostatic pressure, and $\boldsymbol{H}=J \boldsymbol{F}^{-T}$ is the cofactor matrix of $\boldsymbol{F}$.

\section{SPATIAL DISCRETISATION}

In the present work, system (1) will be spatially discretised using the SPH method. Being a mesh-free technique, SPH can be employed effectively in the simulation of high-velocity impacts and high strain-rate deformations. Another advantage of SPH is the locality of the spatial discretisation, with consequent savings in computational power [9]. In a discretised domain, the SPH interpolation at the position $\boldsymbol{x}$ of a quantity $\phi(\boldsymbol{x})$, denoted here as $\langle\phi(\boldsymbol{x})\rangle$, can be approximated by a summation over all neighbouring particles $\boldsymbol{x}_{b}$ :

$$
\langle\phi(\boldsymbol{x})\rangle \approx \sum_{b} V_{b} \phi\left(\boldsymbol{x}_{b}\right) W\left(\boldsymbol{x}_{b}-\boldsymbol{x}, h\right)
$$

where $h$ is the smoothing length, and $W\left(\boldsymbol{x}-\boldsymbol{x}^{\prime}, h\right)$ is usually a polynomial function with compact support ( $W$ vanishes for $\left\|\boldsymbol{x}-\boldsymbol{x}^{\prime}\right\|_{L^{2}} \geq 2 h$ ) for which the following properties are valid:

$$
\int W(\boldsymbol{r}, h) d \boldsymbol{r}=1 ; \quad \lim _{h \rightarrow 0} W(\boldsymbol{r}, h) \rightarrow \delta(\boldsymbol{r})
$$

Calculation of the first derivative of a function $\phi$ in SPH involves the derivation of the kernel function $W$ :

$$
\nabla \phi(\boldsymbol{x})=\sum_{b} V_{b} \phi\left(\boldsymbol{x}_{b}\right) \nabla W\left(\boldsymbol{x}_{b}-\boldsymbol{x}, h\right)
$$

A typical SPH kernel function is presented in fig. $1 \mathrm{~b}$.

The SPH interpolation technique described above performs poorly at and near boundary regions. During the spatial discretisation, the border kernel supports are rendered incomplete by boundary edges, leading to kernel interpolations that are not partitions of unity. To improve the accuracy of the SPH interpolation near boundaries, and to exactly preserve momentum, corrections must be introduced on both the kernel and the kernel gradient [6,7, 10]. The resulting corrected SPH (CSPH) formulation greatly enhances the accuracy and the consistency of the discretisation.

\subsection{CSPH mixed formulation equations}

The mixed $\boldsymbol{p}$ - $\boldsymbol{F}$ system described by equation (1) will be discretised in space using the CSPH particle method with added dissipation from the JST technique ( $\boldsymbol{p}-\boldsymbol{F}$ JST-CSPH scheme). To achieve this, and following the work of Bonet and co-authors [6, 7, 10, 11], the weak statement for the linear momentum evolution must be obtained through the use of work-conjugate principles [1] and integration by parts:

$$
\int_{V} \delta \boldsymbol{v} \cdot \frac{\partial \boldsymbol{p}}{\partial t} d V=\int_{V} \delta \boldsymbol{v} \cdot \rho_{0} \boldsymbol{b} d V+\int_{\partial V} \delta \boldsymbol{v} \cdot \boldsymbol{t}^{B} d A-\int_{V} \boldsymbol{P}: \nabla_{0} \delta \boldsymbol{v} d V
$$




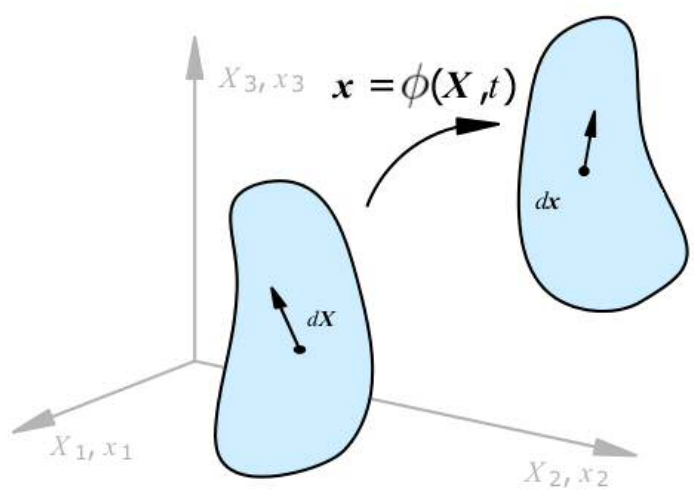

(a)

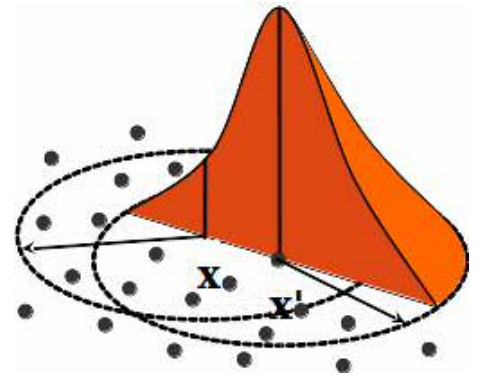

(b)

Figure 1: 1a): The motion $\Phi$ of a continuous solid from a reference configuration $\boldsymbol{X}$ to the current configuration $\boldsymbol{x}$. (1b): Section of a typical, bell shaped SPH kernel function. The contribution of neighbouring particle in $\boldsymbol{x}^{\prime}$ to the computed property at target position $\boldsymbol{x}$ is weighted by the value of its kernel at distance $\left\|\boldsymbol{x}^{\prime}-\boldsymbol{x}\right\|$.

Upon application of the particle integration on the above expression (10), and using the kernel approximation $\nabla_{0} \delta \boldsymbol{v}(\boldsymbol{X})=\sum_{b \in \Lambda_{X}^{b}} V_{b} \delta \boldsymbol{v}_{b} \otimes \nabla_{0} W_{b}(\boldsymbol{X})$ to express the arbitrary virtual velocity field $\delta \boldsymbol{v}$, one obtains:

$$
\frac{d \boldsymbol{p}_{a}}{d t}=\rho_{0} \boldsymbol{b}_{a}+\frac{A_{a}}{V_{a}} \boldsymbol{t}_{a}^{B}+\sum_{b \in \Lambda_{a}^{b}} V_{b}\left(\boldsymbol{P}_{b}+\boldsymbol{P}_{a}\right) \tilde{\nabla}_{0} W_{b}\left(\boldsymbol{X}_{a}\right)
$$

where $V_{b}$ is the volume fraction assigned to the particle in $\boldsymbol{x}_{b}$ and $W_{b}(\boldsymbol{X})$ is the kernel function value there.

Following References [6, 7, 9, -11], the first Piola-Kirchhoff stress tensors $\left\{\boldsymbol{P}_{a}, \boldsymbol{P}_{b}\right\}$ are further approximated by using values of $\left\{\boldsymbol{F}_{a}, \boldsymbol{F}_{b}\right\}$ estimated directly at the particles. In (11), corrections are introduced on the kernel gradient to yield $\tilde{\boldsymbol{\nabla}}_{0} W_{b}\left(\boldsymbol{X}_{a}\right)$, ensuring the correct evaluation of a gradient of a general linear function [6, 10]. However, the above semi-discrete formulation still suffers from the appearance of accumulated numerical instabilities over a long term response. To overcome this, in contrast to the dissipative mechanism introduced by Monaghan [9] through viscous fluxes, here a nodally conservative JST stabilisation $\mathcal{D}\left(\boldsymbol{p}_{a}\right)$ will be incorporated into [11), mirroring CFD techniques [2]:

$$
\frac{d \boldsymbol{p}_{a}}{d t}=\rho_{0} \boldsymbol{b}_{a}+\frac{A_{a}}{V_{a}} \boldsymbol{t}_{a}^{B}+\sum_{b \in \Lambda_{a}^{b}} V_{b}\left(\boldsymbol{P}_{b}+\boldsymbol{P}_{a}\right) \tilde{\nabla}_{0} W_{b}\left(\boldsymbol{X}_{a}\right)+\mathcal{D}\left(\boldsymbol{p}_{a}\right)
$$

The nodally conservative JST stabilisation is additively decomposed into a second order (harmonic) operator $\mathcal{D}_{2}\left(\boldsymbol{p}_{a}\right)$ and a fourth order (biharmonic) operator $\mathcal{D}_{4}\left(\boldsymbol{p}_{a}\right)$ :

$$
\mathcal{D}\left(\boldsymbol{p}_{a}\right)=\mathcal{D}_{2}\left(\boldsymbol{p}_{a}\right)+\mathcal{D}_{4}\left(\boldsymbol{p}_{a}\right)
$$

where 


$$
\begin{aligned}
& \mathcal{D}_{2}\left(\boldsymbol{p}_{a}\right)=\kappa^{(2)} c_{p} h_{\min } \sum_{b \in \Lambda_{a}^{b}} V_{b}\left(\boldsymbol{p}_{b}-\boldsymbol{p}_{a}\right) \tilde{\nabla}_{0}^{2} W_{b}\left(\boldsymbol{X}_{a}\right) \\
& \mathcal{D}_{4}\left(\boldsymbol{p}_{a}\right)=-\kappa^{(4)} c_{p} h_{\min }^{3} \sum_{b \in \Lambda_{a}^{b}} V_{b}\left(\nabla_{0}^{2} \boldsymbol{p}_{b}-\nabla_{0}^{2} \boldsymbol{p}_{a}\right) \tilde{\nabla}_{0}^{2} W_{b}\left(\boldsymbol{X}_{a}\right)
\end{aligned}
$$

In (14) and (15), $c_{p}$ is the pressure wave speed, $h_{\min }$ is the particle spacing, $\kappa^{(2)}$ and $\kappa^{(4)}$ are user-defined parameters and $\tilde{\nabla}_{0}^{2}$ represents the corrected Laplacian operator [6].

In regard to the fibre map evolution (1), the application of particle collocation method [6] directly on (1p), along with the use of kernel approximation $W$, leads to:

$$
\frac{d \boldsymbol{F}_{a}}{d t}=\left.\nabla_{0} \boldsymbol{v}\right|_{a} \approx \sum_{b \in \Lambda_{a}^{b}} \frac{V_{b}}{\rho_{0}}\left(\boldsymbol{p}_{b}-\boldsymbol{p}_{a}\right) \otimes \tilde{\nabla}_{0} W_{b}\left(\boldsymbol{X}_{a}\right)
$$

Finally, the set of stabilised particle equations (12) and (16) can then be explicitly integrated from time step $t^{n}$ to $t^{n+1}$. As described in [1], the scheme is suitably modified to guarantee the conservation of angular momentum.

\section{TEMPORAL DISCRETISATION}

As the resulting set of semi-discrete equations $(12)$ and $(16)$ is not simple, it will be more appropriate to use an explicit type of time integrator. The spatial discretisation discussed above yields a system of ordinary differential equations of the form:

$$
\frac{d \mathcal{U}_{a}}{d t}=\boldsymbol{\mathcal { R }}_{a}\left(\mathcal{U}_{a}, t\right)
$$

where $\boldsymbol{\mathcal { R }}_{a}\left(\mathcal{U}_{a}, t\right)$ represents the right-hand-side of SPH spatial discretisation associated with particle $a$. To advance (17) in time from $t_{n}$ to $t_{n+1}$, an explicit, two-stage TVD-RK time integrator is used:

$$
\left\{\begin{aligned}
\mathcal{U}_{a}^{*} & =\mathcal{U}_{a}^{n}+\Delta t \boldsymbol{\mathcal { R }}_{a}\left(\mathcal{U}_{a}^{n}, t^{n}\right) \\
\mathcal{U}_{a}^{* *} & =\mathcal{U}_{a}^{*}+\Delta t \boldsymbol{\mathcal { R }}_{a}\left(\mathcal{U}_{a}^{*}, t^{n+1}\right) \\
\mathcal{U}_{a}^{n+1} & =\frac{1}{2}\left(\mathcal{U}_{a}^{n}+\mathcal{U}_{a}^{* *}\right)
\end{aligned}\right.
$$

The explicit TVD-RK has been adopted here due to its excellent entropy conserving properties [1, 2]. The time step $\Delta t=t^{n+1}-t^{n}$ is calculated based on the Courant-Friedrichs-Lewy number $\alpha_{C F L}$, by means of $\Delta t=\alpha_{C F L}\left(h / c_{p, \max }\right)$ where $c_{p, \max }$ is the maximum $p$-wave speed and $h$ is the smoothing length.

Along with $\boldsymbol{p}$ and $\boldsymbol{F}$, the geometry, $\boldsymbol{x}$, is also advanced in time using the same time integrator in a monolithic manner.

\section{APPLICATIONS}

A series of benchmark examples are tested in order to assess the applicability and effectiveness of the $\boldsymbol{p}$ - $\boldsymbol{F}$ JST-CSPH mixed algorithm over the Total Lagrangian CSPH displacement based formulation [6, 7, 10]. 
1-D rod in tension A $1 \mathrm{D}$ bar is clamped on one end and left free at the other, where is given an initial velocity of $5 \mathrm{~m} / \mathrm{s}$. The chosen material is linearly elastic, with elastic modulus $E=200 \mathrm{GPa}$ and density $\rho=8000 \mathrm{~kg} / \mathrm{m}^{3}$. The bar is discretised in space using 200 uniformly distributed particles. Aim of this example is to perform a preliminary assessment of the method on a simple problem.

Fig. 2a shows the velocity history at the free tip, while fig. 2b displays the tip displacement. The JST term here has a decisive effect towards achieving a more accurate solution. The Total Lagrangian setting ensures that the simulation does not suffer from tensile instability, a major issue in solid mechanics SPH simulations (see [7, 12, 13]).

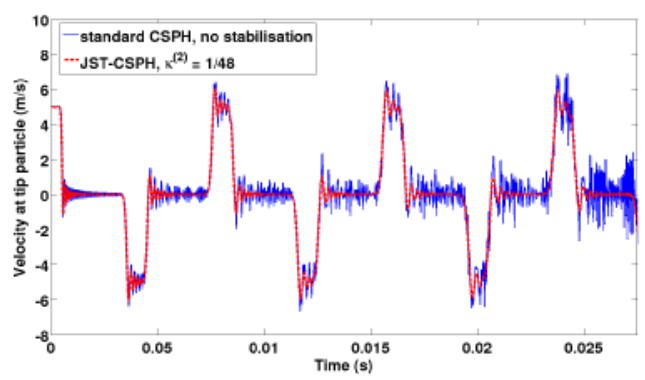

(a) Velocity plot at tip particle with (red) and without (blue) JST dissipation.

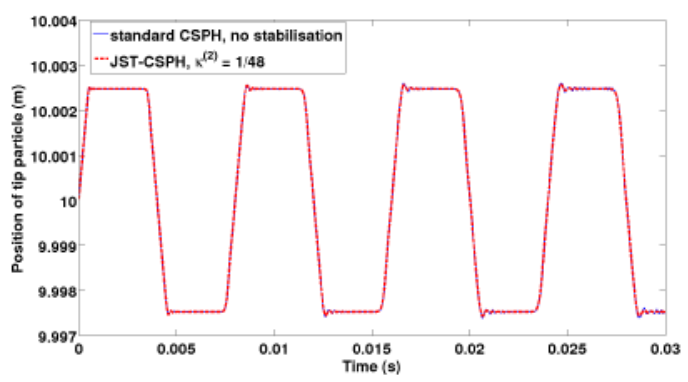

(b) Displacement plot at tip particle with (red) and without (blue) JST dissipation.

Figure 2: 1d tensile test, $\boldsymbol{p}$ - $\boldsymbol{F}$ CSPH based model.

Bending column A bottom end clamp is imposed at $t=0 \mathrm{~s}$ on a 3D column of length $6 \mathrm{~m}$, with unit cross section, initially travelling at constant side velocity $v=10 \mathrm{~m} / \mathrm{s}$. The column is made of a rubbery material modelled as nearly incompressible NeoHookean, with properties $E=17 M P a, \nu=0.45, \rho=1100 \mathrm{~kg} / \mathrm{m}^{3}$. The spatial discretisation is obtained through the CSPH-JST $\boldsymbol{p}-\boldsymbol{F}$ model, using a total of 3969 particles, while a value of $\alpha_{C F L}=0.3$ has been chosen to control the time step size. Fig. 3 demonstrates the performance of the model in a bending dominated scenario.
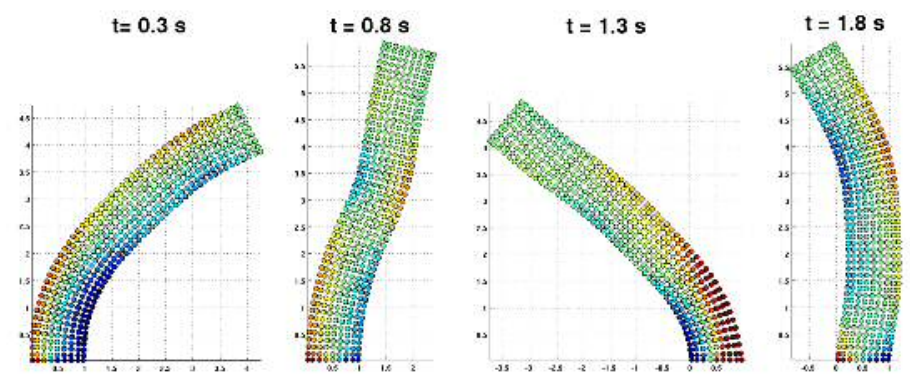

Figure 3: Bending simulation, JST-CSPH $\boldsymbol{p}$ - $\boldsymbol{F}$ based model. NeoHookean material $\left(E=17 M P a, \nu=0.49, \rho=1100 \mathrm{~kg} / \mathrm{m}^{3}\right), 3969$ particles. Pressure plot captured on a cross section of the column at different simulation times.

Twisting column A column with the same geometric and material properties as the one in the previous example is now subjected to a sinusoidal angular velocity of $\boldsymbol{\omega}=100 \sin \left(\frac{\pi z}{2 L}\right) \frac{\boldsymbol{z}}{\|\boldsymbol{z}\|} \mathrm{rad} / \mathrm{s}$ 
and its bottom is clamped. A comparison between fig. 4a (standard CSPH) and fig. 4b ( $\boldsymbol{p}$ - $\boldsymbol{F}$ JST-CSPH) illustrates the robustness of the mixed Total Lagrangian method: while the simulation in fig. $4 a$ fails after a few time steps, the one in fig. $4 \mathrm{~b}$ accurately captures the structure behaviour.
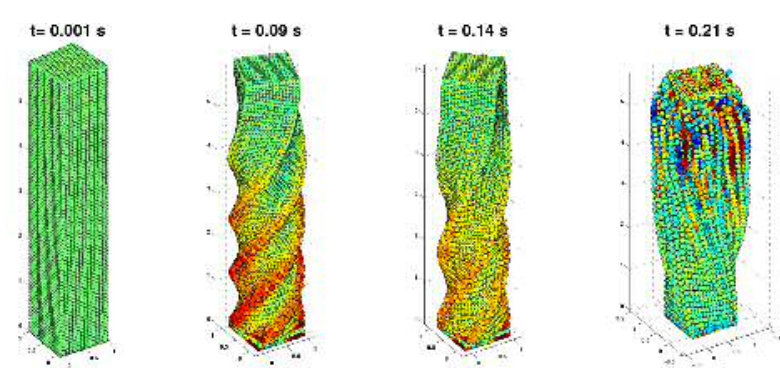

(a) standard CSPH model
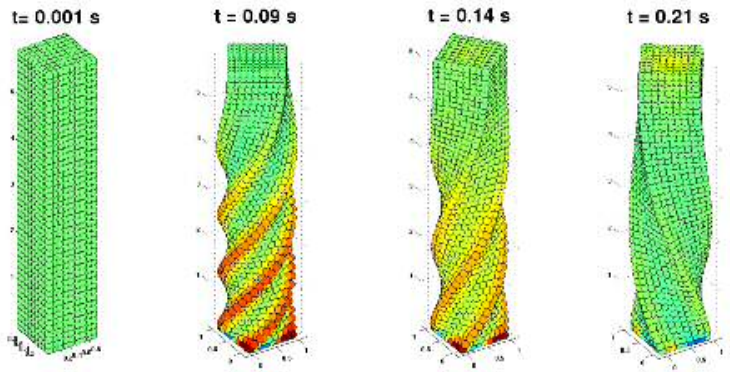

(b) JST-CSPH $\boldsymbol{p}$ - $\boldsymbol{F}$ mixed formulation model

Figure 4: Twisting column, NeoHookean material ( $E=17 M P a, \nu=0.49$, $\rho=1100 \mathrm{~kg} / \mathrm{m}^{3}$ ), 3969 particles. Pressure plot captured at different simulation times.

Punch test A rectangular region of $3 \mathrm{~cm} \times 1 \mathrm{~cm}$ is punched with a constant vertical velocity of $-10 \mathrm{~m} / \mathrm{s}$ on one-third of the top surface. The billet is made of a NeoHookean hyperelastic material with parameters $E=1 M P a, \nu=0.4, \rho=1000 \mathrm{~kg} / \mathrm{m}^{3}$. The particle discretisation has been realised with 225 uniformly distributed particles; the CFL constant has been chosen as $\alpha_{C F L}=0.3$. As reported in [11], the standard CSPH method [6,7, 10] is not capable of solving this problem, which involves extremely large distortions. This deficiency can be alleviated using the proposed JST-CSPH $\boldsymbol{p}$ - $\boldsymbol{F}$ mixed algorithm, as demonstrated in fig. 5 .
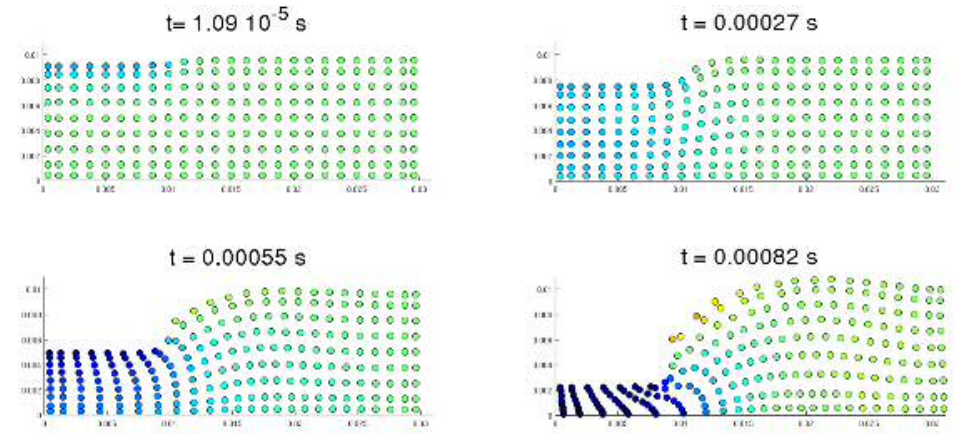

Figure 5: Pressure contour plot for a punch test on a rectangular, 2D billet, captured at various instants of simulation times. Material properties: NeoHookean hyperelastic material, $E=1 \mathrm{MPa}, \nu=0.4, \rho=1000 \mathrm{~kg} / \mathrm{m}^{3}$. JST-CSPH $\boldsymbol{p}$ - $\boldsymbol{F}$ mixed formulation model, 225 particles employed.

Tensile cube A 3-D cube of unit side length is constrained at the bottom face and left free on the other sides. The cube is made of NeoHookean material $(E=21 G P a, \nu=0.3, \rho=$ $7000 \mathrm{~kg} / \mathrm{m}^{3}$ ) and is discretised using 11 particles per edge. It is subjected to a prescribed initial sinusoidal velocity field of $\mathbf{w}=500 \sin \left(\frac{\pi z}{2}\right) \frac{\boldsymbol{z}}{\|\boldsymbol{z}\|} \mathrm{m} / \mathrm{s}$. In the top row of images in fig. 6. JST 
dissipation has not been added to the solution, and chequerboard pressure behaviour develops as the simulation advances in time. To the contrary, as can be observed in the bottom row of fig. 6 , the use of JST-CSPH $\boldsymbol{p}$ - $\boldsymbol{F}$ mixed formulation with $\kappa^{(4)}=1 / 1024$ eliminates the appearance of non-physical pressure fluctuations in near incompressibility regime, stabilising the solution.
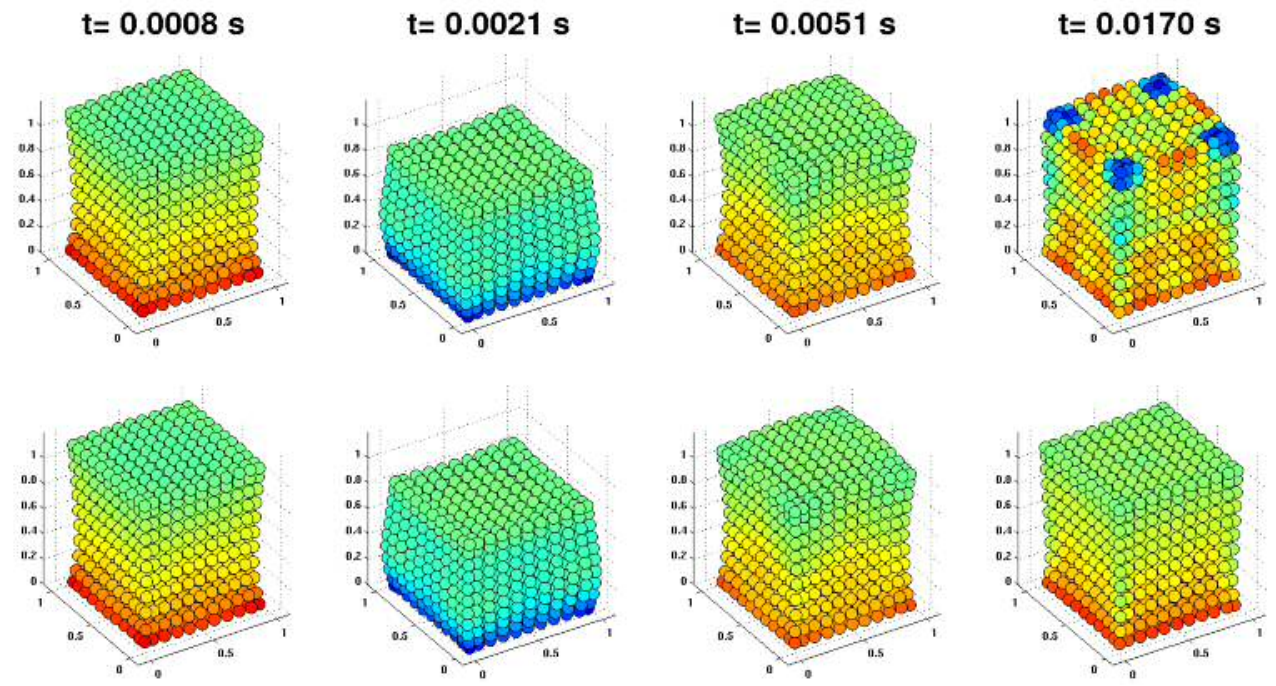

Figure 6: Tensile cube, (top) CSPH $\boldsymbol{p}$ - $\boldsymbol{F}$ mixed formulation, (bottom) JST-CSPH $\boldsymbol{p}$ - $\boldsymbol{F}$ mixed formulation. NeoHookean material $\left(E=21 G P a, \nu=0.3, \rho=7000 \mathrm{~kg} / \mathrm{m}^{3}\right), 1331$ particles. Pressure plot captured at different simulation times.

\section{CONCLUSIONS}

In this work, a stabilised Total Lagrangian mixed formulation $\boldsymbol{p}-\boldsymbol{F}$ JST-CSPH algorithm is presented for the solution of large strain solid dynamics problems. The methodology is based on a system of first order conservation laws, where the linear momentum $\boldsymbol{p}$ and the deformation gradient $\boldsymbol{F}$ are regarded as the primary variables. The well known SPH particle formulation has been modified to accommodate corrections to the kernel interpolating function, resulting in improved behaviour at or near the boundaries of the problem. A nodally conservative JST stabilisation is incorporated into the CSPH $\boldsymbol{p}$ - $\boldsymbol{F}$ mixed formulation algorithm, taking advantage of the conservation laws. The obtained spatial discretisation is combined with a two step TVD Runge-Kutta algorithm for the time integration. Once the numerical model has been so defined, results from numerical simulations of benchmark problems are presented in order to demonstrate the applicability and robustness of the proposed methodology.

\section{ACKNOWLEDGEMENTS}

The authors gratefully acknowledge the financial support provided by the Sêr Cymru National Research Network for Advanced Engineering and Materials, United Kingdom.

\section{REFERENCES}

[1] A.J. Gil, C.H. Lee, J. Bonet and R. Ortigosa. A first order hyperbolic framework for large strain computational solid dynamics. Part II: Total Lagrangian compressible, nearly incompressible and truly incompressible elasticity. CMAME, 300, 146-181, 2016. 
[2] M. Aguirre, A.J. Gil, J. Bonet, A.A. Carreño. A vertex centred Finite Volume JamesonSchmidt-Turkel algorithm for a mixed conservation formulation in solid dynamics. JCP, 259, 672-699, 2014.

[3] C.H. Lee, A.J. Gil, J. Bonet. Development of a stabilised Petrov-Galerkin formulation for conservation laws formulation in Lagrangian fast solid dynamics. CMAME, 268, 40-64, 2014.

[4] C.H. Lee, A.J. Gil and J. Bonet. Development of a cell centred upwind finite volume algorithm for a new conservation law formulation in structural dynamics. $C \& S, 118,13-$ $38,2013$.

[5] I.A. Karim, C.H. Lee, A.J. Gil and J. Bonet. A Two-Step Taylor Galerkin formulation for fast dynamics. $E C, \mathbf{3 1}, 366-387,2014$.

[6] J. Bonet, S. Kulasegaram. Correction and stabilization of smooth particle hydrodynamics methods with applications in metal forming simulations. IJNME, 47, 1189-1214, 2000.

[7] J. Bonet, S. Kulasegaram. Remarks on tension instability of Eulerian and Lagrangian Corrected Smooth Particle Hydrodynamics (CSPH) methods. IJNME, 52, 1203-1220, 2001.

[8] A. Jameson, W.Schmidt, E. Turkel. Numerical Solutions of the Euler Equations by Finite Volume Methods Using Runge-Kutta Time-Stepping Schemes. AIAA 14th Fluid and Plasma Dynamic Conference, AIAA paper 81-1259, Palo Alto, USA, June 1981.

[9] J.J. Monaghan. Smoothed particle hydrodynamics. ARA\&A, 30, 543-574, 1992.

[10] J. Bonet, T.-S.L. Lok. Variational and momentum preservation aspects of Smooth Particle Hydrodynamic formulations. CMAME, 180, 97-115, 1999.

[11] Y. Vidal, J. Bonet and A. Huerta. Stabilized updated Lagrangian corrected SPH for explicit dynamic problems. IJNME, 69, 2687-2710, 2007.

[12] L.D. Libersky, A.G. Petschek, T.C. Carney, J.R. Hipp, F.A. Allahdadi. High strain Lagrangian hydrodynamics. JCP, 109, 67-75, 1993.

[13] J.W. Swegle, D.L. Hicks, S.W. Attaway. Smoothed particle hydrodynamics stability analysis. JCP, 116, 123-134, 1995. 